\title{
Neuroleptic Malignant Syndrome Associated with Valproate in an Adolescent
}

\author{
Veli Yıldııım ${ }^{1}$, Meltem Çobanogulları Direk ${ }^{2}$, Serkan Güneş ${ }^{1}$, Çetin Okuyaz ${ }^{2}$, Fevziye Toros $^{1}$ \\ 'Department of Child and Adolescent Psychiatry, ${ }^{2}$ Department of Pediatric Neurology, Mersin University School of Medicine, Mersin, Turkey
}

\begin{abstract}
Neuroleptic malignant syndrome (NMS) is a life-threatening idiosyncratic reaction that usually occurs after the administration of antipsychotic drugs. Antidepressants, benzodiazepines, and antiepileptic drugs are also suggested to be associated with NMS. It is believed to result from a dopaminergic blockade in the central nervous system. NMS is manifested by hyperthermia, muscle rigidity, autonomic dysfunction, altered mental status, leukocytosis, and elevated serum creatinine phosphokinase. Valproate is commonly used in the treatment of many psychiatric and neurologic disorders. Valproate can precipitate NMS, especially when used with antipsychotic drugs concurrently. A 17-year-old male patient, who presented with fever, muscular rigidity, confusion, sweating, and tachycardia was admitted to the emergency room. He had been taking only valproate for the last two months for bipolar disorder. His laboratory analyses revealed raised serum hepatic enzymes, creatinine phosphokinase, and myoglobin levels. Considering fever, rigidity, autonomic dysfunction, cognitive alteration, and high creatinine phosphokinase levels, the patient was diagnosed with NMS. In this paper, we aim to discuss the association between valproate and NMS.
\end{abstract}

KEY WORDS: Neuroleptic malignant syndrome; Valproate; Adolescent; Child.

\section{INTRODUCTION}

Neuroleptic malignant syndrome (NMS) was first recognized in 1956 by Ayd but was only defined in 1960 by Delay and Deniker. ${ }^{1)}$ NMS is a rarely encountered, life threatening complication that usually appears within the first two weeks of antipsychotic therapy. ${ }^{2)}$ Moreover, NMS cases due to certain antidepressants, benzodiazepines, antiepileptic drugs, metoclopramide, and lithium have also been reported. ${ }^{3)}$ Its incidence ranges from $0.02 \%$ to $3 \%$ with a mortality rate around $10 \%{ }^{4}{ }^{4}$ The most common causes of death include rhythm disorders, respiratory failure, and renal or cardiovascular insufficiency. ${ }^{5)}$

NMS is clinically characterized by fever, muscular rigidity, autonomic dysfunction, and altered mental state, whereas its laboratory features are comprised of elevated serum hepatic enzymes, creatinine phosphokinase, and white blood cell count. ${ }^{6}$ Dopamine receptor blockage is thought to play a role in the pathogenesis of NMS. ${ }^{7)}$ The risk factors for NMS include high-dose antipsychotic use, rapid dose titration, using the parenteral form of anti-

\footnotetext{
Received: February 12, 2016 / Revised: March 16, 2016

Accepted: April 15, 2016

Address for correspondence: Serkan Güneş, MD

Department of Child and Adolescent Psychiatry, Mersin University School of Medicine, Çiftlikköy Kampüsü, Yenişehir, Mersin, Turkey Tel: +90-5542587368, Fax: +90-3242410092

E-mail:dr_sgunes@hotmail.com
}

psychotic drugs, advanced age, male sex, malnutrition, dehydration, traumatic or organic cerebral injury, iron deficiency, infections, and concurrent use of lithium, anticholinergic agents, and certain antidepressants. ${ }^{5)}$

The present paper aims to discuss NMS developed in an adolescent, who had been diagnosed with bipolar disorder and receiving valproate. Informed consent was obtained from the patient's parents for publication of this case report.

\section{CASE}

A 17-year-old male patient was admitted to the emergency room with complaints of fever, sweating, sore throat, loss of appetite, weakness, lethargy, and reduced speech. He was diagnosed with type I bipolar disorder two years ago and valproate, haloperidol, and biperiden therapies were commenced. Haloperidol and biperiden therapies were tapered two months prior to the emergency room visit and that only valproate therapy continued for the last two months. He had been taking $500 \mathrm{mg}$ valproate per day for two years. The patient's body temperature was high for the last week and remained high all day long. His mental state was completely altered and he was not eating, speaking, sleeping, or leaving his room. There was no substance or other drug use or surgical intervention in his history.

Examination of the patient revealed moderate general

(c) This is an Open-Access article distributed under the terms of the Creative Commons Attribution Non-Commercial License (http://creativecommons.org/licenses/by-nc/4.0) which permits unrestricted non-commercial use, distribution, and reproduction in any medium, provided the original work is properly cited. 
status. His heart rate was 110 beats/minute, respiratory rate was 22 times/minute, blood pressure was $110 / 70 \mathrm{mmHg}$, and axillary body temperature was $38.5^{\circ} \mathrm{C}$. Confusion, distractibility, and extensive muscular rigidity were evident on his neurological examination. Mental examination revealed incorrect orientation. His affect was dysphoric, anxious, and irritable. He had visual and hearing hallucinations. Other system examinations were unremarkable.

Results of laboratory analyses were as follows: glucose, $154 \mathrm{mg} / \mathrm{dl}$ (60-100 mg/dl); urea, $78 \mathrm{mg} / \mathrm{dl}$ (10.7-38.5 $\mathrm{mg} / \mathrm{dl})$; creatinine, $1.08 \mathrm{mg} / \mathrm{dl}$ (0-0.9 mg/dl); ammonium, $84 \mu \mathrm{mol} / \mathrm{L}$ (36-85 $\mu \mathrm{mol} / \mathrm{L}) ;$ lactate, $13.1 \mathrm{mg} / \mathrm{dl}$ (4.5-19.8 $\mathrm{mg} / \mathrm{dl})$; Na, $149 \mathrm{mEq} / \mathrm{L}$ (132-145 mEq/L); K, $4.7 \mathrm{mEq} / \mathrm{L}$ (3.1-5.1 mEq/L); Cl, $116 \mathrm{mEq} / \mathrm{L}$ (96-111 mEq/L); Ca, 8.9 $\mathrm{mg} / \mathrm{dl}(8.4-10.9 \mathrm{mg} / \mathrm{dl})$; creatinine phosphokinase, $>4,267$ $\mathrm{U} / \mathrm{L}$ (0-145 U/L); alanine aminotransferase, $70 \mathrm{U} / \mathrm{L}$ (0-39 $\mathrm{U} / \mathrm{L})$; aspartate aminotransferase, $240 \mathrm{U} / \mathrm{L}$ (0-47 U/L); gamma-glutamyl transferase, $20 \mathrm{U} / \mathrm{L}(0-17 \mathrm{U} / \mathrm{L})$; lactate dehydrogenase, $759 \mathrm{U} / \mathrm{L}(0-580 \mathrm{U} / \mathrm{L})$; myoglobin, $>1,000$ $\mathrm{ng} / \mathrm{ml}(25-58 \mathrm{ng} / \mathrm{ml})$; thyroid stimulating hormone, 1.59 $\mu \mathrm{IU} / \mathrm{ml}(0.15-3.5 \mu \mathrm{IU} / \mathrm{ml})$; free T4, $15.34 \mathrm{pmol} / \mathrm{L}(10-25$ pmol/L). In complete blood count, leukocyte count was $9,610 / \mathrm{mm}^{3}\left(4,500-13,000 / \mathrm{mm}^{3}\right)$, hemoglobin was $15.2 \mathrm{~g} / \mathrm{dl}$ $(12-15 \mathrm{~g} / \mathrm{dl})$ and hematocrit was $44.6 \%$ (34-43\%). Serum valproate concentration was $<12.5 \mu \mathrm{g} / \mathrm{ml}(50-100 \mu \mathrm{g} / \mathrm{ml})$.

The patient was admitted to the pediatric neurology clinic for further investigation. Peripheral blood, throat, and urine cultures were performed; there was no growth. Procalcitonin and C-reactive protein were studied and found to be within the normal limits. Computed cranial tomography was reported to be normal, and no abnormality in arterial blood gas analysis was observed. Cerebrospinal fluid examination, complete urinalysis, and chest X-ray were unremarkable. Electrocardiogram showed sinus tachycardia.

The patient was diagnosed with NMS, because it was the only diagnosis by which all the clinical and laboratory findings could be explained.

\section{DISCUSSION}

Although NMS is usually observed within the first ten days of antipsychotic drug use, NMS may appear at any phase of treatment, independent of duration and dose. ${ }^{2)}$ Many late-onset NMS cases have been reported in the literature. ${ }^{8-10)}$ There was a case of a patient developing NMS after ten years of antipsychotic drug use. ${ }^{11)}$

Although the clinical signs are variable, muscular rigidity, autonomic dysfunction, and cognitive changes are the main symptoms. Frequent laboratory findings include elevated creatinine phosphokinase, leukocytosis, and myoglobulinuria. ${ }^{6)}$ The Diagnostic and Statistical Manual of Mental Disorders, fourth edition (DSM-IV) diagnostic criteria, which are created by the American Psychiatry Association, can be used to diagnose NMS. Diagnosis of NMS according to the DSM-IV can be made in the presence of both of major symptoms (high fever and muscular rigidity) and at least two or more of minor symptoms (sweating, dysphagia, chilling, incontinence, cognitive alteration, mutism, tachycardia, elevated or variable blood pressure, leukocytosis, or laboratory findings suggestive of muscular injury). ${ }^{6}$ In the present case, the patient met the two major (fever and rigidity) and five of the minor (confusion, sweating, tachycardia, mutism, and elevated creatinine phosphokinase) criteria.

Head trauma, malignant hyperthermia, drug intoxication, central nervous system diseases, central anticholinergic syndrome, pheochromocytoma, thyroid storm, and sepsis should be considered in the differential diagnosis of NMS. ${ }^{6}$ In the present case, other intracranial pathologies were excluded based on anamnesis, physical examination, and computed tomography. Malignant hyperthermia was not considered since $\mathrm{PaCO}_{2}$ was not elevated in the arterial blood gas analysis and there was no anesthetic or neuromuscular blocking agent use in the patient's history. Serum valproate level was $<12.5 \mu \mathrm{g} / \mathrm{ml}$; hence, drug intoxication was excluded. Anticholinergic syndrome was excluded because of the absence of anticholinergic drug use and sepsis was excluded because of absence of growth in blood, throat, or urine cultures. Thyroid pathologies were eliminated due to normal thyroid hormone concentrations.

In general, typical antipsychotic drugs cause NMS due to their high antidopaminergic effects (D2 receptor blockade). However, even an atypical antipsychotic with low antidopaminergic effects may cause NMS. ${ }^{12)}$ Moreover, it has been reported that many drugs without known antidopaminergic effects have also caused NMS. Therefore, some factors, besides D2 receptor blockade, are considered to be responsible for the pathogenesis of NMS. ${ }^{7}$ Benzodiazepines, tricyclic antidepressants, serotonin reuptake inhibitors, metoclopramide, amphetamine, lithium, and tetrabenazine may also cause NMS. ${ }^{3)}$ Antiepileptic drugs such as carbamazepine, oxcarbazepine, and lamotrigine were also demonstrated to be associated with NMS. ${ }^{13-15)}$

Valproate is commonly used as an anticonvulsant and mood stabilizer in neurologic and psychiatric disorders. ${ }^{16)}$ Studies of valproate have mostly focused on its mechanisms of action on the GABAergic system. Specifically, 
valproate has been shown to increase whole-brain GABA levels and potentiate response by inhibiting GABA-transaminase and activating glutamic acid decarboxylase. Valproate blocks voltage-dependent sodium channels, and it is also known to function as a weak blocker of T-type calcium channels. ${ }^{17)}$ In a case report, Verma et al. ${ }^{3)}$ reported that NMS developed following the addition of valproate in the treatment of a patient receiving antipsychotic drugs for two years, without remarkable complication. Tanii et al ${ }^{18)}$ reported a case, in which a patient developed NMS due to withdrawal of levomepromazine from their treatment, while continuing treatment with valproate. In many studies stating that antipsychotics cause NMS, patients had been receiving concurrent valproate therapy. ${ }^{7,19,20)}$

We hypothesized that valproate might induce NMS via the GABA system in our patient. Although the exact mechanism of how NMS is connected with the GABA system is not yet entirely understood, it is known that the GABAergic medications, baclofen, and lamotrigine may cause symptoms of NMS. ${ }^{15,21)}$ It was also reported that chronic treatment of rodents with valproate increased the brain dopamine levels in some areas and decreased it in other brain areas. ${ }^{17)}$ Changes in the dopaminergic system due to chronic valproate therapy might be another mechanism that induced NMS symptoms in our case. In addition, when the patient in the present case used haloperidol two month ago, it constituted hypodopaminergic and D2 supersensitive statement. This statement might be induced by the addition of valproate via one of the possible mechanisms, and predispose the patient to NMS.

Although valproate does not directly cause NMS, it seems to be an agent associated with NMS development in the present case. To our knowledge, this is the first case of NMS, in an adolescent, associated with valproate treatment.

\section{- Acknowledgments}

This article was conducted at the Mersin University Medical Faculty.

\section{REFERENCES}

1. Védie C, Poinso F, Hemmi F, Rivet B. Major symptoms and differential diagnosis of neuroleptic malignant syndrome: three case reports. Eur Psychiatry 2000;15:334-337.

2. Fekadu A, Bisson JI. Neuroleptic malignant syndrome: diagnostic and therapeutic dilemmas. Behav Neurol 2005;
16:9-13.

3. Verma R, Junewar V, Rathaur BPS. An atypical case of neuroleptic malignant syndrome precipitated by valproate. BMJ Case Rep 2014. doi: 10.1136/bcr-2013-202578.

4. Sarkar P, Natarajan C, Gode N. Prevalence of neuroleptic malignant syndrome in 672 consecutive male in-patients. Indian J Psychiatry 2009;51:202-205.

5. Rasmussen KG. Risk factors for neuroleptic malignant syndrome. Am J Psychiatry 1998;155:1639-1640.

6. Strawn JR, Keck PE Jr, Caroff SN. Neuroleptic malignant syndrome. Am J Psychiatry 2007;164:870-876.

7. Ananth J, Parameswaran S, Gunatilake S, Burgoyne K, Sidhom T. Neuroleptic malignant syndrome and atypical antipsychotic drugs. J Clin Psychiatry 2004;65:464-470.

8. Kern JL, Cernek PK. Delayed risperidone-induced neuroleptic malignant syndrome. Ann Pharmacother 1996;30:300.

9. Lee MS, Lee HJ, Kim L. A case of delayed NMS induced by risperidone. Psychiatr Serv 2000;51:254-255.

10. Kunz M, Gomes FA, Tramontina JF, Kapczinski F. Lateonset neuroleptic malignant syndrome in a patient using olanzapine. J Clin Psychopharmacol 2007;27:303-304.

11. Oglodek E, Szota A, Araszkiewicz A. Olanzapine-induced neuroleptic malignant syndrome after 10 years of treatment. Aust N Z J Psychiatry 2013;47:972.

12. Chakraborty N, Johnston T. Aripiprazole and neuroleptic malignant syndrome. Int Clin Psychopharmacol 2004;19: 351-353.

13. Nisijima K, Kusakabe Y, Ohtuka K, Ishiguroa T. Addition of carbamazepine to long-term treatment with neuroleptics may induce neuroleptic malignant syndrome. Biol Psychiatry 1998;44:930-931.

14. Angelopoulos P, Markopoulou M, Kyamidis K, Bobotas K. Neuroleptic malignant syndrome without fever after addition of oxcarbazepine to long-term treatment with amisulpride. Gen Hosp Psychiatry 2008;30:482-484.

15. Ishioka M, Yasui-Furukori N, Hashimoto K, Sugawara N. Neuroleptic malignant syndrome induced by lamotrigine. Clin Neuropharmacol 2013;36:131-132.

16. Bae KY, Jang JE, Kim YH, Kim JM, Yoon JS. Valproateinduced hyperammonemic encephalopathy caused by free carnitine deficiency in a patient with bipolar disorder: a case report. Clin Psychopharmacol Neurosci 2009;7:57-62.

17. Johannessen CU, Johannessen SI. Valproate: past, present, and future. CNS Drug Rev 2003;9:199-216.

18. Tanii H, Saka K, Inoue K, Okada M. Neuroleptic malignant syndrome following levomepromazine discontinuation. J Neuropsychiatry Clin Neurosci 2010;22:E16.

19. Gortney JS, Fagan A, Kissack JC. Neuroleptic malignant syndrome secondary to quetiapine. Ann Pharmacother 2009; 43:785-791.

20. Ladds B, Thomas P, Mejia C, Hauser D. Extreme elevation of creatinine phosphokinase levels in neuroleptic malignant syndrome associated with atypical antipsychotics. Am J Psychiatry 2009;166:114-115.

21. Samson-Fang L, Gooch J, Norlin C. Intrathecal baclofen withdrawal simulating neuroleptic malignant syndrome in a child with cerebral palsy. Dev Med Child Neurol 2000; 42:561-565. 